\title{
Temperature-Dependent and Bistable Current-Voltage Measurements in Zinc Porphyrin Molecular Junctions
}

\author{
Swatilekha Saha, $^{\dagger}$ Adrien Nicolai, $^{\dagger}$ Jonathan R. Owens, $^{\dagger}$ Alexandra Krawicz, ${ }^{\ddagger}$ Peter H. Dinolfo, ${ }^{*}{ }^{\ddagger}$ \\ Vincent Meunier, ${ }^{*},, \S$ and Kim M. Lewis ${ }^{*} \dagger$
}

${ }^{\dagger}$ Department of Physics, Applied Physics, and Astronomy, ${ }^{\ddagger}$ Department of Chemistry and Chemical Biology, and ${ }^{\S}$ Department of Materials Science and Engineering, Rensselaer Polytechnic Institute, Troy, New York 12180, United States

Supporting Information

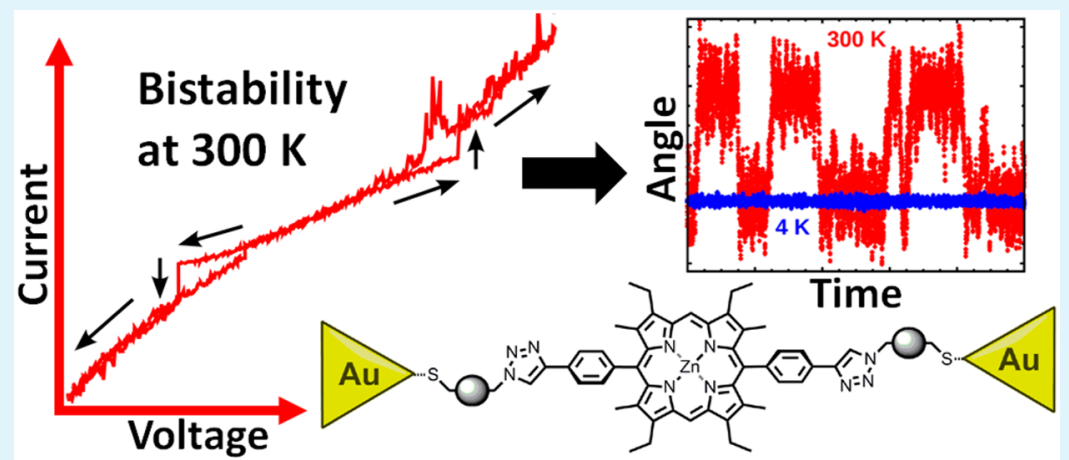

ABSTRACT: We report bistability in current-voltage curves from di(PEP)PorZn in an electromigrated molecular junction. Bistability was observed at $\pm 0.3 \mathrm{~V}$ at $300 \mathrm{~K}$ but did not occur at $4 \mathrm{~K}$. No bistability was identified at $300 \mathrm{~K}$ for another porphyrin molecule (di(Xyl)PorZn), where the phenyl-ethnyl-phenyl (PEP) side groups were replaced with a flexible $p$-xylene. Molecular dynamics simulations show that bistability may be due to conformation changes related to the fluctuation of the dihedral angle surrounding the zinc and/or the rotation of the porphyrin central plane of the molecule. Results suggest that other mechanisms may play a role in the current-voltage characteristics observed.

KEYWORDS: temperature dependence, bistability, electromigration, molecular junctions, porphyrin, molecular dynamic simulations

M olecular electronics are an attractive option for decreasing the size of devices to nanometer scale. Because only a few electrons contribute to the conductance of these devices, the overall power consumption is reduced compared to conventional electronic devices. Additionally, molecules can offer a range of functionalities similar to existing microelectronics. For example, the electrical properties of molecules can be used to build a variety of electronic components, such as transistors, rectifiers, and amplifiers. ${ }^{1-3}$ Another promising application is the use of molecules to mimic electrical switches. Upon applying an electric field, it has been found that some molecules can change from a high to a low conductance state due to the bistability of the molecule. ${ }^{4-7}$ Although most of these studies have been performed using a scanning tunneling microscope (STM), very few studies have examined bistability in a two-terminal device at room temperature. Fabricating and effectively controlling the behavior of these molecular switches requires a fundamental understanding of the electronic transport across a metalmolecule-metal junction and of the origins of the bistability of the molecules. ${ }^{8-13}$

In this study, we investigated the transport properties of a highly conjugated aromatic system of porphyrin molecules with a zinc $(\mathrm{Zn})$ atom ligated to the central porphyrin: $\operatorname{di}(\mathrm{Xyl})$ -
PorZn (Figure 1a) and di(PEP)PorZn (Figure 1b). The molecules were synthesized using $\mathrm{Cu}(\mathrm{I})$-catalyzed azidealkyne cycloaddition, or "click chemistry" starting materials in the formation of the final complex (see Supporting Information (SI)). This method leads to a highly synthetic yield of porphyrin and may allow for rapid modification of the side linkers. A single or a few molecules of $\mathrm{di}(\mathrm{Xyl})$ PorZn or $\mathrm{di}(\mathrm{PEP})$ PorZn were positioned in a $2-3$ $\mathrm{nm}$ gap ${ }^{15}$ created by electromigration. Gold nanowires of $\sim 50$ $\mathrm{nm}$ width and $200 \mathrm{~nm}$ length with $5 / 25 \mathrm{~nm}$ of titanium/gold $(\mathrm{Ti} / \mathrm{Au})$ were fabricated on a silicon substrate with $100 \mathrm{~nm}$ thermally grown oxide using electron beam lithography. Larger contact pads $(1500 \mu \mathrm{m} \times 1500 \mu \mathrm{m})$ with $5 / 100 \mathrm{~nm}$ of $\mathrm{Ti} / \mathrm{Au}$ for wire bonding were made by photolithography. After the liftoff process was completed using acetone, the porphyrin molecules were deposited on the nanowires by placing the samples in a $0.01 \mathrm{mM}$ solution for $12 \mathrm{~h}$. Electromigration was completed in a Class 100 clean room to form $\sim 2-3 \mathrm{~nm}$ gaps. ${ }^{15}$ From the device geometry and because the molecule size is

Received: March 19, 2015

Accepted: April 28, 2015

Published: April 28, 2015 
(a)

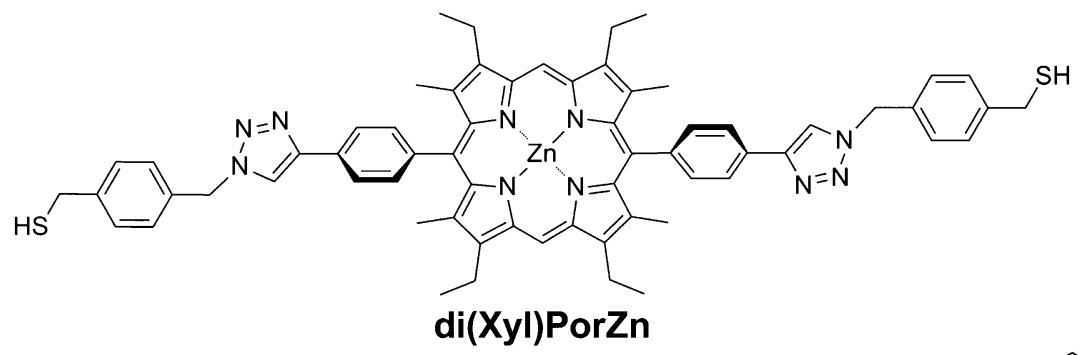

(b)

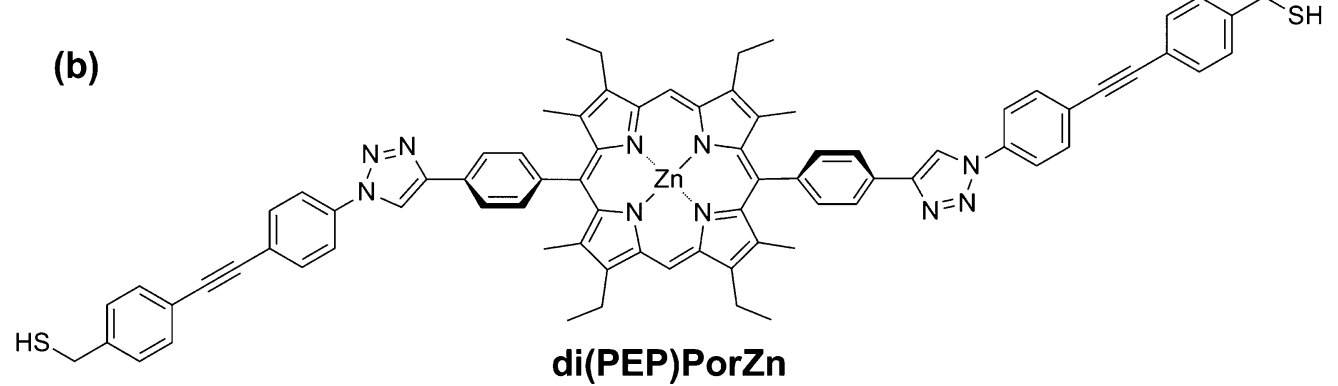

Figure 1. Schematic of zinc porphyrin molecules: (a) di(Xyl)PorZn and (b) di(PEP)PorZn.
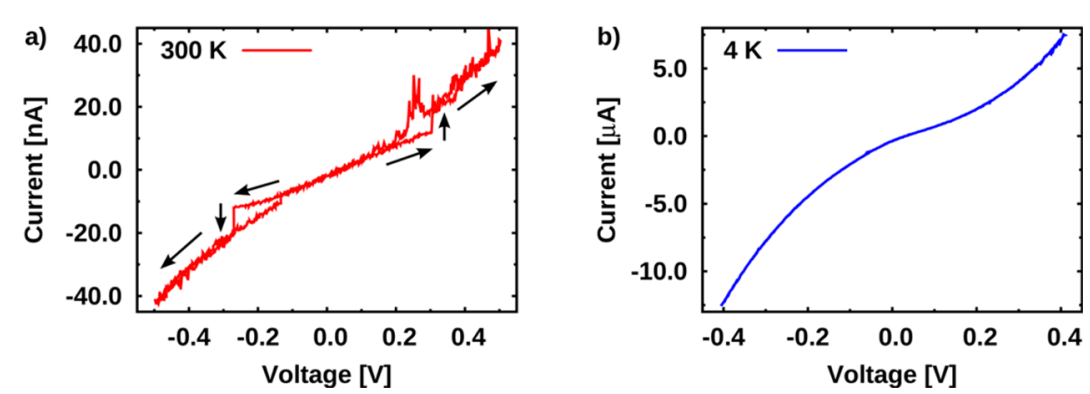

Figure 2. Typical $I-V$ characteristics from di(PEP)PorZn in an electromigrated nanogap. (a) At $300 \mathrm{~K}$, bistability was observed at $\pm 0.3 \mathrm{~V}$, and (b) at $4 \mathrm{~K}$, no bistability was observed. The voltage sweep was $0 \rightarrow-0.5 \rightarrow 0.5 \rightarrow 0(\mathrm{~V})$.
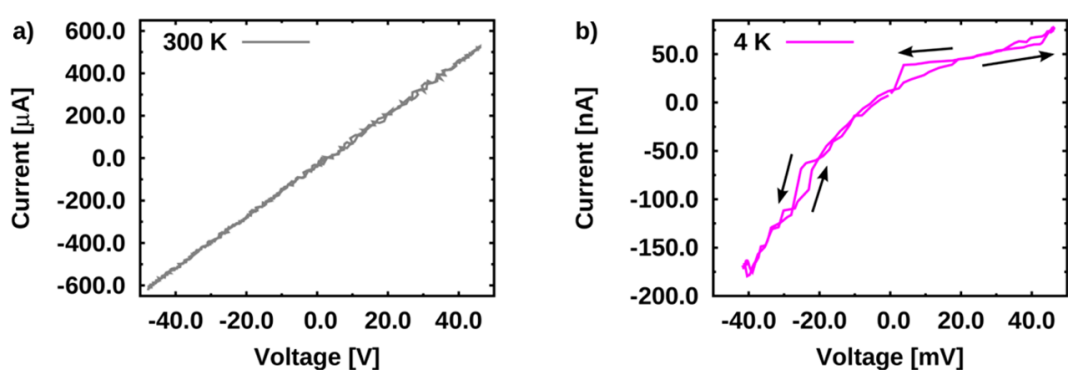

Figure 3. Typical $I-V$ characteristics from di(Xyl)PorZn in an electromigrated nanogap. (a) $I-V$ curve measured at $300 \mathrm{~K}$ and (b) bistability observed near 5 and $-30 \mathrm{mV}$ at $4 \mathrm{~K}$. The voltage sweep was $0 \mathrm{~V} \rightarrow-40 \mathrm{mV} \rightarrow 40 \mathrm{mV} \rightarrow 0 \mathrm{~V}$.

$\sim 4.8 \mathrm{~nm}$, we expect a single or at most a few molecules will bridge the gap forming the molecular junction. Currentvoltage $(I-V)$ curves were measured by applying a direct current (DC) bias to the sample, and the current was measured by a Keithley digital multimeter.

At $300 \mathrm{~K}$, we observed bistability in $I-V$ curves from di(PEP)PorZn molecular junctions formed by electromigrated nanogaps. A characteristic $I-V$ curve is shown in Figure 2a. The voltage was swept from $0 \rightarrow-0.5 \rightarrow 0.5 \rightarrow 0(\mathrm{~V})$. The curve shows bistability near $\pm 0.3 \mathrm{~V}$. We found that near $0.3 \mathrm{~V}$ the current increased and then decreased near $-0.3 \mathrm{~V}$. Bistability was observed for four molecular junctions tested at $300 \mathrm{~K}$ that exhibited measurable current. However, for these devices, the bistability did not occur at the same voltage values previously mentioned. However, all samples showed an increase to a higher current at a positive bias and a decrease to a lower current at a negative bias (see SI). A similar behavior was observed by Qiu et al. for $\mathrm{Zn}$ (II) etioporphyrin I. $^{16} \mathrm{We}$ attribute the bistability at different voltage values to the fact that each electromigrated nanogap may not be identical in size because the latter can vary from 2 to $3 \mathrm{~nm}$. Therefore, for each nanogap a different electric field exists across the junction (the importance of electric field will be discussed below). Bistability was always observed between -1 and $1 \mathrm{~V}$. Molecular junctions with $\operatorname{di}(\mathrm{PEP})$ PorZn were also tested at $4.2 \mathrm{~K}$. A characteristic $I-V$ curve is shown in Figure $2 \mathrm{~b}$. From the same molecular junction tested, at least five additional $I-V$ curves were measured (see SI). None of the curves exhibited bistability. All of the curves were measured within a voltage range from -0.5 to $0.5 \mathrm{~V}$. 
a)
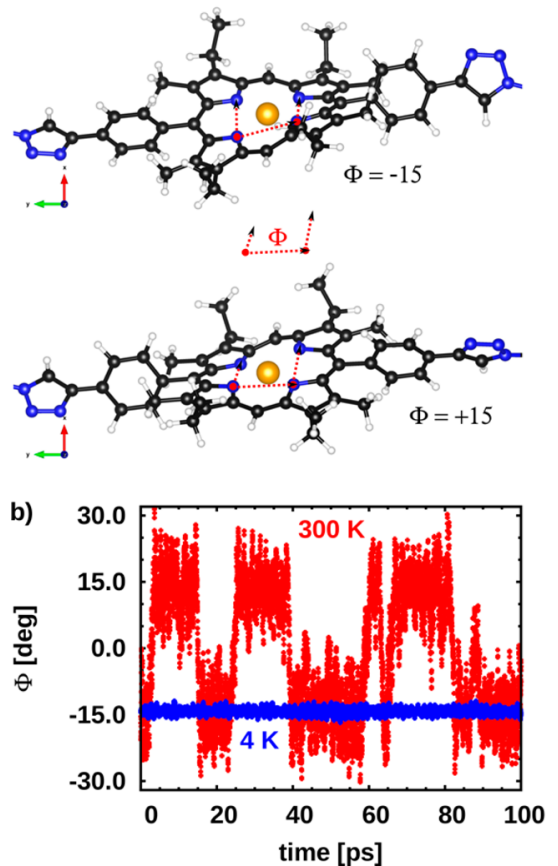

c)
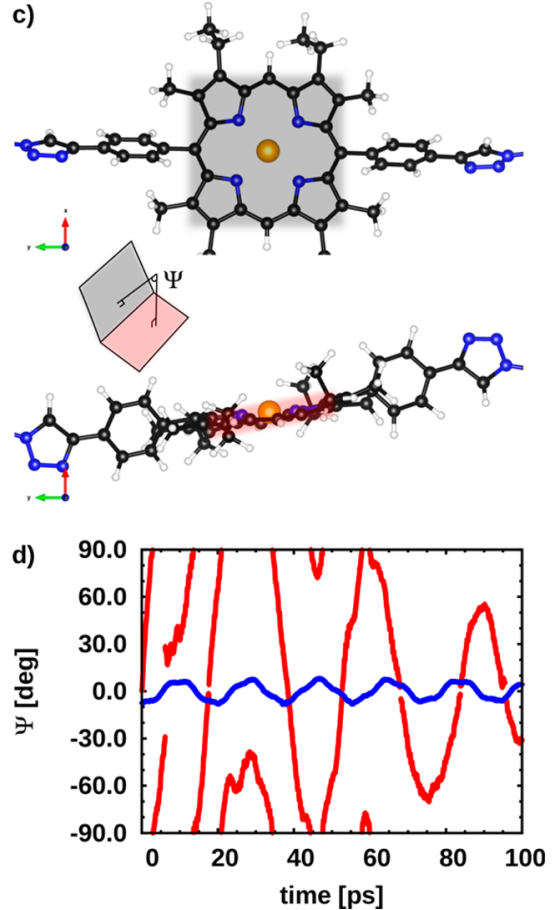

Figure 4. (a, b) Local conformational changes corresponding to the fluctuation of the dihedral angle $\Phi$ made by the 4 nitrogen atoms surrounding the $\mathrm{Zn}$ atom for di(PEP)PorZn. (c, d) Global conformational changes corresponding to the rigid rotation of the porphyrin central plane of the molecule composed of the carbon and nitrogen atoms as highlighted for $\operatorname{di}(\mathrm{PEP})$ PorZn. The angle $\Psi$ represents the angle between the normal vectors of the plane at time $t-1$ and $t$.

To better understand the bistability of the porphyrin molecular junctions, we measured $I-V$ characteristics at $4.2 \mathrm{~K}$ of $\operatorname{di}(\mathrm{Xyl})$ PorZn. Compared to di(PEP)PorZn, this structure lacks the rigid, aromatic phenyl-ethnyl-phenyl (PEP) side groups. Instead, a more flexible $p$-xylene linker was added also using "click chemistry" (see SI). The voltage range was applied from -40 to $40 \mathrm{mV}$. The bias voltage was kept low to protect the sample from further electromigration. At $300 \mathrm{~K}$, the $I-V$ curve was measured, and no bistability was observed (see Figure $3 \mathrm{a})$. In Figure $3 \mathrm{~b}$, bistability appeared at $4 \mathrm{~K}$ in the $\mathrm{I}-\mathrm{V}$ curve at 5 and $-30 \mathrm{mV}$. The voltage sweep was repeated several times. After the third $I-V$ sweep, the bistability remained only in the positive bias range and disappeared in the negative bias range (see SI). For these results, we did not investigate the cause of the disappearance of the bistability. However, it is suspected that successive sweeps of the voltage may have changed the molecular junction. Besides bistability or conformational changes of the molecule that may be responsible for this behavior, the disappearance of bistability could be due to hybridization changes at the molecule-metal interface $^{17}$ or contact geometries at the molecule-metal interface. ${ }^{18}$ Conductance curves showed changes in the slope corresponding to the bistability observed in the $I-V$ curve (see SI).

Molecular dynamics (MD) simulations were performed to explain the bistability observed in the porphyrin molecular junctions. Self-consistent charge density functional tight binding (SCC-DFTB) MD simulations were carried out using the DFTB-plus 1.2 software package. ${ }^{19,20}$ Geometry optimizations were performed using the conjugate gradient algorithm as implemented in DFTB-plus using a force criterion of $0.01 \mathrm{eV} /$ $\AA$. The set of parameters used for the simulations are the following: the mio package $\mathrm{e}^{20,21}$ for carbon, oxygen, nitrogen, hydrogen, and sulfur atoms and the znorg package ${ }^{22}$ for the $\mathrm{Zn}$ atom of the porphyrin molecule. The time step used in all simulations was $1 \mathrm{fs}$, and the coordinates of all of the atoms were saved every $10 \mathrm{fs}$. The initial velocities were chosen randomly according to a Maxwell-Boltzmann distribution. The temperature was kept at the desired value using a NoséHoover thermostat ${ }^{23,24}$ with a coupling strength of 3127.6 $\mathrm{cm}^{-1}$, which corresponded to the highest vibrational mode frequency of the system. ${ }^{25}$ We used a tolerance of $10^{-7}$ for the SCC loop calculations with an Anderson mixer parameter of 0.5 .

In the present calculations, we do not consider the gold $(\mathrm{Au})$ electrodes. We assume that porphyrin molecules are attached to the two electrodes by fixing the two terminal parts, which contain the sulfur $(\mathrm{S})$ atoms bound to $\mathrm{Au}\left(10\right.$ atoms, $\mathrm{CH}_{3}-\mathrm{CO}$ $\left.\mathrm{S}-\mathrm{CH}_{2}\right)$. MD runs of $100 \mathrm{ps}$ in a vacuum for the di(PEP)PorZn molecule were performed at two different temperatures $(T=4$ and $300 \mathrm{~K}$ ) with an applied voltage of $500 \mathrm{mV}$ to reproduce the experimental procedure. To do so, an electric field $E_{y}=-V / L_{y}$ is applied, where $L_{y}$ is the length of the molecule initially aligned along the $y$ axis. In addition, another MD run of 100 ps at $T=300 \mathrm{~K}$, but without applied voltage, was performed for the di(PEP)PorZn molecule. Finally, an MD run at $T=4 \mathrm{~K}$ and $V=500 \mathrm{mV}$ was performed for the $\mathrm{di}(\mathrm{Xyl})$ PorZn molecule.

The two porphyrin molecules ( $\mathrm{di}(\mathrm{Xyl})$ PorZn and di(PEP)PorZn) investigated experimentally were studied. From MD simulations, the local (Figure 4a) and global (Figure 4c) conformational dynamics of $\mathrm{di}(\mathrm{PEP})$ PorZn were investigated to determine an explanation for the bistability at $300 \mathrm{~K}$. Figure $4 \mathrm{~b}$ (red curve) shows the changes in the local conformation corresponding to the fluctuations of the dihedral angle $(\Phi)$ made by the four nitrogen atoms surrounding the zinc atom. At $300 \mathrm{~K}$, two distinct events were observed at $-15^{\circ}$ and $15^{\circ}$. In 

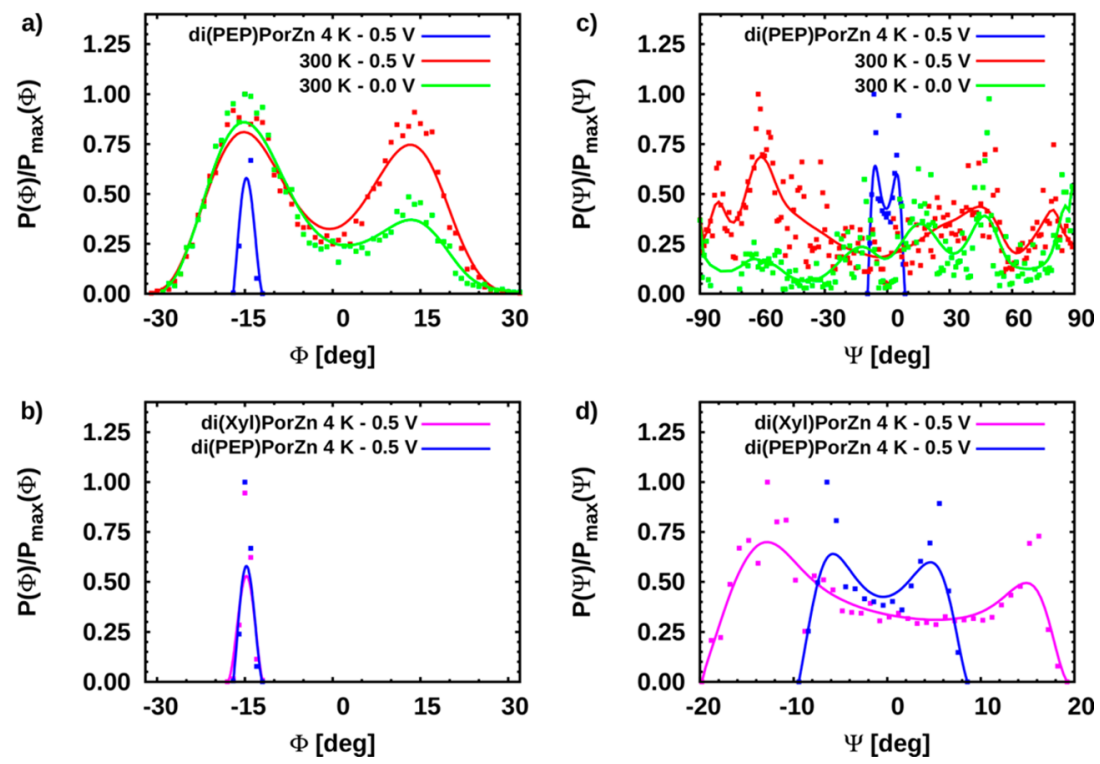

Figure 5. Probability distribution function $(P)$ of the dihedral angle $\Phi$ and the angle $\Psi$ between the porphyrin planes computed from MD simulations of di(Xyl)PorZn and di(PEP)PorZn molecules. (a) $P(\Phi)$ computed for di(PEP)PorZn molecules at $4 \mathrm{~K}$ (blue) and $300 \mathrm{~K}$ (red) with an applied voltage of $0.5 \mathrm{~V}$ and at $300 \mathrm{~K}$ with no voltage (green). (b) $P(\Phi)$ computed for di(Xyl)PorZn (magenta) and di(PEP)PorZn (blue) at $4 \mathrm{~K}$ with an applied voltage of $0.5 \mathrm{~V}$. (c) $P(\Psi)$ computed for di(PEP)PorZn molecules at $4 \mathrm{~K}$ (blue) and $300 \mathrm{~K}$ (red) with an applied voltage of $0.5 \mathrm{~V}$ and $300 \mathrm{~K}$ with no voltage (green). (d) $P(\Psi)$ computed for di(Xyl)PorZn (magenta) and di(PEP)PorZn (blue) at $4 \mathrm{~K}$ with an applied voltage of 0.5 V. For clarity, all of the distributions have been normalized to the maximum value $P_{\max }$.

contrast, at $4 \mathrm{~K}$, there was no change in the dihedral angle over the time span of 100 ps (blue curve in Figure 4b). Figure $4 d$ shows the global conformational changes corresponding to the rotation of the porphyrin central plane of the molecule. The plane is made of the carbon and nitrogen atoms, as highlighted in Figure 4c. The angle $(\Psi)$ associated with this rotation represents the angle between the normal vectors of the plane at time $t-1$ and $t$. Similarly, there was very little fluctuation in the rotation of the porphyrin at $4 \mathrm{~K}$ (blue curve in Figure $4 \mathrm{~d}$ ). However, as shown in Figure $4 \mathrm{~d}$ (red curve), there were large fluctuations in the angle $\left(-90^{\circ}\right.$ to $\left.90^{\circ}\right)$ for di(PEP)PorZn. These local and global conformations account for the bistability (and lack thereof) observed in the $I-V$ curves shown in Figure 2.

To investigate the relationship between the structural changes of the porphyrin and the experimental $I-V$ measurements, we performed a statistical study of the conformational changes as a function of experimental parameters. This study investigated changes in temperature $T$ for a given applied voltage $V$ (Figure $5 \mathrm{a}$ and $\mathrm{c}$ ) and vice versa for the di(PEP)PorZn as well as the difference between the two molecules di(Xyl)PorZn and $\operatorname{di}(\mathrm{PEP})$ PorZn at the same conditions for $T$ and $V$ (Figure $5 \mathrm{~b}$ and $\mathrm{d}$ ). Statistical analyses of the porphyrin conformational changes are shown using the probability distribution function $(P)$ with $\mathrm{MD}$ simulations for the characteristic angles $\Phi$ and $\Psi$.

First, we investigated the local conformational change. Figure 5a shows $P(\Phi)$ for the di(PEP)PorZn molecule as a function of $T$ and $V$. Similar to the angle trajectories shown in Figure $4 \mathrm{~b}$, $P(\Phi)$ at $4 \mathrm{~K}$ shows one peak near $\Phi=-15$, whereas $P(\Phi)$ at $300 \mathrm{~K}$ shows two peaks near $\Phi=-15$ and +15 , which have the same probability. The initial configuration in our $\mathrm{MD}$ simulation of $\mathrm{di}(\mathrm{PEP})$ PorZn was $\Phi=-15$; however, by symmetry, we would observe the same behavior starting from $\Phi=+15$. At $T=300 \mathrm{~K}$ and without applied voltage $V$, we observed a statistical difference for $P(\Phi)$. Although $P(\Phi)$ shows two peaks at $\Phi=-15$ and +15 for $V=0.5 \mathrm{~V}$, the two peaks are no longer equiprobable for $V=0$, indicating that the local conformational change depends on the applied voltage. However, this result is due to finite MD simulations, and this observation will not hold at much longer simulation times. We extended our analysis to the local conformational change for $\operatorname{di}(\mathrm{Xyl})$ PorZn at $4 \mathrm{~K}$, which showed bistability in the experimental $I-V$ curve (Figure $3 \mathrm{~b}$ ). As shown in Figure $5 \mathrm{~b}$, $P(\Phi)$ is characterized by a single peak, as is the case for the di(PEP)PorZn molecule at $4 \mathrm{~K}$. This observation indicates that the local conformational change around the $\mathrm{Zn}$ atom was not the only reason for the experimental observation and/or points to different mechanisms between the $\operatorname{di}(\mathrm{Xyl})$ PorZn and di(PEP)PorZn molecules.

Second, we investigated the global conformational change using the same procedure as described earlier. Figure $5 \mathrm{c}$ shows $P(\Psi)$ for the $\operatorname{di}($ PEP)PorZn molecule as a function of $T$ and $V$. As shown in Figure $4 d$, the rotation of the porphyrin plane is restricted to angles changing between -10 and +10 at $4 \mathrm{~K}$, whereas at $300 \mathrm{~K}, \Psi$ can take all values between -90 and +90 . In addition, $P(\Psi)$ at $T=300 \mathrm{~K}$ shows higher probabilities for $|\Psi|$ at large values $(\sim 60)$ compared to values around zero. At $V$ $=0, P(\Psi)$ shows the same distribution for $V=0.5 \mathrm{~V}$. The only minor change corresponds to a larger probability around the value of zero for $V=0$ than for $V=0.5 \mathrm{~V}$. By comparing $P(\Psi)$ for $\mathrm{di}(\mathrm{Xyl})$ PorZn and di(PEP)PorZn at $4 \mathrm{~K}$ as shown in Figure $5 \mathrm{~d}$, we observed that the nature of the molecule has a large influence on the global conformational change and could be the reason why we experimentally observe bistability in the $I-V$ curve at $4 \mathrm{~K}$. For $\mathrm{di}(\mathrm{Xyl})$ PorZn at $4 \mathrm{~K}, \Psi$ can take values between -20 and 20, which is two times larger than the values for di(PEP)PorZn at the same conditions for $T$ and $V$.

On the basis of our studies, we conclude that the global conformational change observed in the MD simulations of the di(Xyl)PorZn and di(PEP)PorZn molecules seems to be a plausible explanation for the experimental bistability because it 
is observed for both molecules at different $T$. However, according to our simulations, it is unlikely to be the only reason, and the local conformational change observed for the di(PEP)PorZn molecule could be another explanation. This implies that the mechanism for bistability can be slightly different for the two porphyrin systems studied. Another explanation to be considered is that the conformational changes observed in the MD simulations can be directly related to the existence of phonons of relatively low frequencies $(\omega<600$ $\mathrm{cm}^{-1}$ ), which correspond to large amplitudes for the normal modes of the molecules (see SI). The transport phenomenon across porphyrin molecules could excite phonons and therefore contribute to the conformational changes and bistability observed experimentally in the $I-V$ curves.

We investigated the current voltage characteristics of di(PEP)PorZn and di(Xyl)PorZn confined in an electromigrated nanogap. Bistability was observed for di(PEP)PorZn at $300 \mathrm{~K}$, which did not appear at $4 \mathrm{~K}$. These results were rationalized using $\mathrm{MD}$ simulations, which showed two distinct bistable events at $300 \mathrm{~K}$ for di(PEP)PorZn related to the local conformation dynamics of the dihedral angle made of the four nitrogen atoms surrounding the $\mathrm{Zn}$ atom. We also found that there is a relationship between the conformation change and the applied bias voltage for $\mathrm{di}(\mathrm{PEP})$ PorZn. For di(Xyl)PorZn, $I-V$ curves showed that bistability at $300 \mathrm{~K}$ did not exist, but at $4 \mathrm{~K}$, the angle between the porphyrin planes is a possible explanation for the bistability. We also suggest that low frequency phonons can contribute to structural changes and bistability in the porphyrin systems. These investigations provide insight into the electron transport behavior of porphyrin molecules in an electromigrated nanogap for the design of molecular devices.

\section{ASSOCIATED CONTENT}

\section{S Supporting Information}

Details on chemical synthesis of the porphyrins: di(PEP)PorZn and $\operatorname{di}(\mathrm{Xyl})$ PorZn. Current-voltage characteristics from di(PEP)PorZn and di(Xyl)PorZn, including differential conductance plots for di(Xyl)PorZn, and calculated spectra of the conformational changes as a function of phonons in the porphyrins. The Supporting Information is available free of charge on the ACS Publications website at DOI: 10.1021/ acsami.5b02449.

\section{AUTHOR INFORMATION}

\section{Corresponding Authors}

*E-mail: dinolp@rpi.edu.

*E-mail: meuniv@rpi.edu.

*E-mail: lewisk2@rpi.edu.

\section{Notes}

The authors declare no competing financial interest.

\section{ACKNOWLEDGMENTS}

This work was partially supported by National Science Foundation Grant DMR-1150866 and the National Science Foundation National Nanotechnology Infrastructure Network (NNIN). All calculations were performed at the Center for Computational Initiative at RPI. A.N. and V.M. were supported by the Office of Naval Research. This material is based upon work supported by Rensselaer Polytechnic Institute and the National Science Foundation under DGE-0333314 and CHE1255100 .

\section{REFERENCES}

(1) Diez-Perez, I.; Li, Z. H.; Hihath, J.; Li, J. H.; Zhang, C. Y.; Yang, X. M.; Zang, L.; Dai, Y. J.; Feng, X. L.; Muellen, K.; Tao, N. J. GateControlled Electron Transport in Coronenes as a Bottom-up Approach Towards Graphene Transistors. Nat. Commun. 2010, 1, $1-5$.

(2) Tao, N. J. Electron Transport in Molecular Junctions. Nat. Nanotechnol. 2006, 1, 173-181.

(3) Cheng, J.-F.; Zhou, L.; Liu, M.; Yan, Q.; Han, Q.; Gao, L. TipContact Related Low-Bias Negative Differential Resistance and Rectifying Effects in Benzene-Porphyrin-Benzene Molecular Junctions. J. Chem. Phys. 2014, 141, 174304.

(4) Sharma, P.; Fursina, A.; Poddar, S.; Ducharme, S.; Gruverman, A. Coplanar Switching of Polarization in Thin Films of Vinylidene Fluoride Oligomers. Appl. Phys. Lett. 2014, 105, 182903.

(5) Moresco, F.; Meyer, G.; Rieder, K. H.; Tang, H.; Gourdon, A.; Joachim, C. Conformational Changes of Single Molecules Induced by Scanning Tunneling Microscopy Manipulation: A Route to Molecular Switching. Phys. Rev. Lett. 2001, 86, 672-675.

(6) Alemani, M.; Peters, M. V.; Hecht, S.; Rieder, K. H.; Moresco, F.; Grill, L. Electric Field-Induced Isomerization of Azobenzene by Scanning Tunneling Microscopy. J. Am. Chem. Soc. 2006, 128, 1444614447.

(7) Wu, S. W.; Ogawa, N.; Nazin, G. V.; Ho, W. Conductance Hysteresis and Switching in a Single-Molecule Junction. J. Phys. Chem. C 2008, 112, 5241-5244.

(8) Getty, S. A.; Engtrakul, C.; Wang, L.; Liu, R.; Ke, S. H.; Baranger, H. U.; Yang, W.; Fuhrer, M. S.; Sita, L. R. Near-Perfect Conduction through a Ferrocene-Based Molecular Wire. Phys. Rev. B 2005, 71, 241401.

(9) Jo, M. H.; Grose, J. E.; Baheti, K.; Deshmukh, M. M.; Sokol, J. J.; Rumberger, E. M.; Hendrickson, D. N.; Long, J. R.; Park, H.; Ralph, D. C. Signatures of Molecular Magnetism in Single-Molecule Transport Spectroscopy. Nano Lett. 2006, 6, 2014-2020.

(10) Park, J.; Pasupathy, A. N.; Goldsmith, J. I.; Chang, C.; Yaish, Y.; Petta, J. R.; Rinkoski, M.; Sethna, J. P.; Abruna, H. D.; McEuen, P. L.; Ralph, D. C. Coulomb Blockade and the Kondo Effect in Single-Atom Transistors. Nature 2002, 417, 722-725.

(11) Yoon, D. H.; Lee, S. B.; Yoo, K. H.; Kim, J.; Lim, J. K.; Aratani, N.; Tsuda, A.; Osuka, A.; Kim, D. Electrical Conduction through Linear Porphyrin Arrays. J. Am. Chem. Soc. 2003, 125, 11062-11064.

(12) Liang, W. J.; Shores, M. P.; Bockrath, M.; Long, J. R.; Park, H. Kondo Resonance in a Single-Molecule Transistor. Nature 2002, 417, 725-729.

(13) Yu, H. B.; Luo, Y.; Beverly, K.; Stoddart, J. F.; Tseng, H. R.; Heath, J. R. The Molecule-Electrode Interface in Single-Molecule Transistors. Angew. Chem., Int. Ed. 2003, 42, 5706-5711.

(14) Kolb, H. C.; Finn, M. G.; Sharpless, K. B. Click Chemistry: Diverse Chemical Function from a Few Good Reactions. Angew. Chem., Int. Ed. 2001, 40, 2004-2021.

(15) Saha, S.; Qian, G.; Lewis, K. M. Fabrication of Nanogaps by a Progressive Electromigration Technique Using Wires of Various Thicknesses. J. Vac. Sci. Technol., B 2011, 29, 061802.

(16) Qiu, X. H.; Nazin, G. V.; Ho, W. Mechanisms of Reversible Conformational Transitions in a Single Molecule. Phys. Rev. Lett. 2004, 93, 196806

(17) Moore, A. M.; Dameron, A. A.; Mantooth, B. A.; Smith, R. K.; Fuchs, D. J.; Ciszek, J. W.; Maya, F.; Yao, Y. X.; Tour, J. M.; Weiss, P. S. Molecular Engineering and Measurements to Test Hypothesized Mechanisms in Single Molecule Conductance Switching. J. Am. Chem. Soc. 2006, 128, 1959-1967.

(18) Quek, S. Y.; Kamenetska, M.; Steigerwald, M. L.; Choi, H. J.; Louie, S. G.; Hybertsen, M. S.; Neaton, J. B.; Venkataraman, L. Mechanically Controlled Binary Conductance Switching of a SingleMolecule Junction. Nat. Nanotechnol. 2009, 4, 230-234.

(19) Aradi, B.; Hourahine, B.; Frauenheim, T. Dftb+, a Sparse Matrix-Based Implementation of the Dftb Method. J. Phys. Chem. A 2007, 111, 5678-5684. 
(20) Elstner, M.; Porezag, D.; Jungnickel, G.; Elsner, J.; Haugk, M.; Frauenheim, T.; Suhai, S.; Seifert, G. Self-Consistent-Charge DensityFunctional Tight-Binding Method for Simulations of Complex Materials Properties. Phys. Rev. B 1998, 58, 7260-7268.

(21) Niehaus, T. A.; Elstner, M.; Frauenheim, T.; Suhai, S. Application of an Approximate Density-Functional Method to Sulfur Containing Compounds. J. Mol. Struct. THEOCHEM 2001, 541, 185194.

(22) Moreira, N. H.; Dolgonos, G.; Aradi, B.; da Rosa, A. L.; Frauenheim, T. Toward an Accurate Density-Functional TightBinding Description of Zinc-Containing Compounds. J. Chem. Theory Comput. 2009, 5, 605-614.

(23) Nose, S. A Unified Formulation of the Constant Temperature Molecular-Dynamics Methods. J. Chem. Phys. 1984, 81, 511-519.

(24) Hoover, W. G. Canonical Dynamics - Equilibrium Phase-Space Distributions. Phys. Rev. A 1985, 31, 1695-1697.

(25) Martyna, G. J.; Tuckerman, M. E.; Tobias, D. J.; Klein, M. L. Explicit Reversible Integrators for Extended Systems Dynamics. Mol. Phys. 1996, 87, 1117-1157. 